\title{
Synthesis of Nanoparticles and Its Uses in Dentistry- A Review
}

\author{
Aashritha Shenava ${ }^{1}$, S. M. Sharma ${ }^{2}$, Veena Shetty ${ }^{3}$, Shama Rao ${ }^{4}$, Vishak R. ${ }^{5}$
}

\begin{abstract}
${ }^{1}$ Department of Prosthodontics, AB Shetty Institute of Dental Sciences, Mangalore, Karnataka, India. ${ }^{2}$ Department of Oral Surgery, AB Shetty Institute of Dental Sciences, Mangalore, Karnataka, India. ${ }^{3}$ Department of Microbiology, K. S. Hegde Medial Academy, Ullal, Karnataka, India. ${ }^{4}$ Nitte University Center for Stem Cells, Mangalore, Karnataka, India. ${ }^{5}$ Central Research Laboratory KSHEMA, Deralakatte, Mangalore, Karnataka, India.
\end{abstract}

\section{ABSTRACT}

\section{BACKGROUND}

The control of chemical composition of the surface of nanoparticles is an important factor for the reliability and reproducibility of the nanoparticles. The control of chemical composition is paramount due to the extremely small size they have and due to a high surface area to volume ratio. Another factor is the interaction between the size of individual nanoparticles and host matrix materials. The crucial factor is that while adding the nanoparticle, the performance of the material into which they are incorporated is enhanced. Among the methods of synthesis, the physical and chemical methods are explained in detail in this article. The actions of the dental materials are enhanced by nanoparticles. There can be a molecular scale interaction between these nanoparticles and the dental materials comprising the matrix. This could be due to size of individual nanoparticles which often approaches that of the host matrix materials. The use of nanoparticles addresses the aforementioned difficulty by combining high mechanical strength with long-term polish retention in the dental materials. In order to increase the cohesive strength of dental adhesives it is common to use filler particles which have been treated with a polymerizable silane. Nanoparticles have excellent wear resistance in nanofill composite compared to other restoratives materials. The wear resistance of dental composites is very important. Wear may result from physical or pathological conditions. Several invitro test protocols to accelerate and predict the wear of dental materials have been developed. Typically hybrid composites have clinically shown excellent wear resistance. Nanoparticles of barium sulphate and ytterbium fluoride have been added to glass ionomers to improve their radiopacity. This article deals with the various methods of synthesis and this factor has been used in dental materials without adversely affecting other properties. Nanoparticles are a boon to science and technology.

\section{KEY WORDS}

Nanoparticles, Synthesis
Corresponding Author: Dr. Aashritha Shenava, Department of Prosthodontics, AB Shetty Institute of Dental Sciences, Mangalore, Karnataka, India. E-mail: draashrithashenava@nitte.edu.in

DOI: $10.14260 / j e m d s / 2019 / 780$

Financial or Other Competing Interests: None.

How to Cite This Article:

Shenava A, Sharma SM, Shetty $V$, et al. Synthesis of nanoparticles and its uses in dentistry- a review. J. Evolution Med. Dent. Sci. 2019;8(48):3613-3616, DOI: $10.14260 /$ jemds $/ 2019 / 780$

Submission 21-06-2019, Peer Review 13-11-2019, Acceptance 20-11-2019, Published 02-12-2019. 


\section{BACKGROUND}

Using various physical and chemical methods, functional materials can be synthesized in nanoscale.[1] The US national nanotechnology has three requirements for nanotechnology1. Technology development at an atomic, molecular, or macromolecular levels, in the length scale of 1-100 nm range. 2. Creating and using devices, structures, and system that will have novel properties and functions because of their small and -or intermediate size. 3. Ability to control or manipulate on the atomic- molecular scale.

To control the ultimate performance of the material to which the nanoparticles are incorporated is crucial thus control of the chemical composition of the surface of nanoparticles becomes a prerequisite to the reliability and reproducibility of the nanoparticles. There can be a molecular scale interaction between these nanoparticles and the materials comprising the matrix. This could be due to size of individual nanoparticles often approaches that of the host matrix materials.

Nanotechnology is used in most of the medical field in high degree of planning. They are incorporated because they manipulate the structure of materials to provide dramatic improvements in chemical, mechanical, and optical properties. This factor has been utilized in providing unique characteristics to dental adhesives such as adhesion strength and radiopacity without adversely affecting other properties.

\section{Synthesis of Nanoparticles}

Nanoparticles can be synthesized using bottom-up and topdown methods. In the top-down approach lithography is used to remove unwanted regions on the thin films and it is finally etched to get nanostructures. This procedure is used in advanced electronic industries. Contrary to the top -down approach the bottom-up approach involves mostly synthesis from molecular or atomic level. These methods have been used in industries and the success is dependent on the requirements of the application.

\section{Synthesis by Mechanical Attrition}

Large coarse -grained particles are ground to form smaller sized particles with the help of ceramic processing and powder metallurgy. These attrition techniques which is a type of "top down" manufacturing technique have been used to produce nanoparticles. The procedure involves consumption of energy and is successful in providing nanostructured components for dental materials.[2]

\section{Synthesis through Sol-Gel Process}

This method is considerably less cost effective comparatively to the other techniques of synthesis. A sol is defined as a fluid suspension of a colloidal solid in a liquid. A gel is a semi-solid colloidal suspension of a solid dispersed in a liquid.[3] A sol Gel preparative method which is in a liquid form is mixed with different precursor which gives a solution.

\section{Functionalization of Oxide Nanoparticles}

Two types of functionalization of the surface of nanoparticles are followed. In the first method, the nanoparticle or nanocluster is isolated and grafted to the nanoparticle. The organic group is used in the second method in the synthesis. There is a reaction between the oxide nanoparticles and silicon halides. The modification after synthesis is seen by using silane coupling agent.

\section{Synthesis of Silsesquioxane Nanoparticles}

These type of nanoparticles are like cage structures, ladder and random structures. The silsesquioxane have the empirical formula $\mathrm{RSiO}_{1.5}$. They have hybrid compositions where the ratios of oxygen to silicon are different. They are polyhedral and often referred to as POSS materials.

\section{Synthesis of Polymer-Templated Nanoparticles}

Assemblies of polymers are used to template the synthesis of nanoparticles. These assemblies create an environment suitable for the synthesis and are made of polymers.[4] Nanoapetite needle like crystals are synthesized by polyacrylic acid, surfactants are also used as templates as they produce micelles.[5,6] Chitosan and collagen precipitate Nano hydroxyapatite crystals. All these polymer -Template nanoparticles are used in dental adhesives.[7]

Dentistry is one field where nanoparticles like nanocomposites containing oxide nanoparticles are used to enhance the properties of the material. Nanohybrids and nanofills are the widely used dental nano composites In nanohybrids, the particles are large and are 4-5 $\mathrm{nm}$ in size. In nanofills, the particles vary from 1 to $100 \mathrm{~nm}$ throughout the resin matrix. Resin Based dentistry has been one of the breakthroughs in adhesive dentistry. Adhesive dentistry provides cosmetically accepted restorations along with conservation of tooth structure with potential tooth reinforcement. However, over the years composite materials have been able to meet all the requirements of both posterior and anterior restorations and provided with excellent shade selection. ${ }^{[18]}$ The microfill composite were the restorative material of choice for anterior teeth and hybrid composite materials are used for posterior teeth as they are considerably having high strength and high wear resistance. [9] The hybrid restoration material did not have enough polish retention which is needed for anterior restorations. The microfill restorative material had superior polish retention but decreased strength and wear resistance. By the advent of nanoparticles both the properties such as polish retention and mechanical strength was enhanced in a single material.

\section{Nanofill Composites}

The nanofills consist of two types of nanoparticles monodispersed non-aggregated form of silica and nanometric agglomerated particles with the filler particle size ranging from 1-100 nm.[10] Prevention of agglomeration is done by adding a coupling agent such as methacrylopropyl trimethoxysilane (MPTS).The mode of reaction is by the silica ester which is added to one end of the bonding to the inorganic surface and methacrylate group to the other end, this prevents agglomeration.[11,12] Researchers at $3 \mathrm{M}$ have introduced nanofillers called nanoclusters which help in improving the rheological properties. The nanoclusters range from 5 to $75 \mathrm{~nm}$ and are usually from oxides of silica and zirconia. They have an average size of $0.6 \mathrm{ps}$ to $10 \mathrm{~nm}$. 


\section{Nanohybrid Composites}

Agglomeration usually causes undesirable rheological properties and in order to overcome this aspect the manufacturers have improvised by adding prepolymerised organic filler which are called nanohybrids. The nanohybrids still have certain drawbacks such as loss of initial gloss.[13]

\section{Silsesquioxane-Based Composites}

It is seen that $5 \%$ methacrylate functionalized POSS decreased the shrinkage drastically and improved the mechanical properties and optical properties.[14] Studies have been reported by Soh et al that incorporation of silsesquioxane have decreased the shrinkage.[15,16]

\section{Calcium Phosphate and Calcium Fluoride Nanoparticles- Based Composites}

$\mathrm{Xu}$ and coworkers have used nanoparticles with calcium fluoride and calcium phosphate as they release phosphate and calcium ions.[17,18,19] The new advances with nanoparticles was the introduction of nanohydroxyapatite (HAP) which mimic human enamel and studies are in the process of completion. Nano silica fused whiskers were added to enhance the mechanical properties.[20,21,22]

\section{Nanoparticles in Glass lonomer Systems}

Resin Modified Glass Ionomers (RMGI) are important as they release fluoride which helps in the remineralisation of tooth, which helps in decreasing the sensitivity of the teeth. Zirconia nanoclusters are introduced as the resin modified glass ionomers have low aesthetic property.[23,24,25,26] The advantage over the conventional RMGI is the opacity of the zirconia monomers is very low and this gives a realistic effect. Radio opacity is improved by adding ytterbium fluoride and barium sulphate. Nanofluorapetite mixed with fluoroaluminosilicate powder enhanced the mechanical properties.[27,28,29,30]

\section{Nanotechnology in Dental Adhesives}

The filler particles are added to dental adhesion while storage tend to settle down. The filler particles enhances the cohesive strength. Silane treated nanoparticles of silica and zirconia were added to overcome the disadvantage of filler particles.[31] Thus the nanoparticles are too small to settle down and uniformly mix in the adhesive material. $3 \mathrm{M}^{\mathrm{TM}}$ ESPETM, single bond Plus and 3M ${ }^{\mathrm{TM}}$ ESPETM $^{\mathrm{TM}}$, Scotchbond ${ }^{\mathrm{TM}} \mathrm{SE}$ adhesives are best examples.[32]

\section{Selected Properties of Dental Materials Containing Nano Particles}

Optical Properties- The most important feature of using nanoparticles is the improved mechanical strength, wear resistance and extremely low visual opacity due to the small particle size. The nanoparticles are smaller than the wavelength of visible light. Although the nanofill composite have an average size which is similar to that of microhybrid fillers or conventional hybrid the nanoclusters are different from hybrid and micro hybrid filler particles. The nanoclusters consist of spacing between and the addition of nanomer reduces the spacing and gives a smooth finish in the nanofilled composite. The surface of hybrid and microhybrid restorations wear away in contrast to the nanoclusters. Thus the filler particles are usually exposed in case of hybrid and microhybrid restorations and wear causes formation of voids and pits thus leaving an unpolished surface.

Wear Properties- Wear could be due to masticatory load or neuromuscular habits such as bruxism which leads to attrition of the restoration.[33] Tests have been conducted and hybrid composite showed wear resistance which was developed by Academisch Cantrum Tandhulkund Amsterdam. [34] AFM (Atomic Farce Microscopy ) was used to find the wear resistance and Filtek Superior had better wear resistance than most of the hybrid and microhybrid composites.[35] Studies by Cha et al have proven wear resistance of nanofill composite using an oral simulator.[36]

Mechanical Properties- Curtis et al studied the flexural strength of nanosized filler particles and nanoclusters. The biaxial flexural strength was more with nanofill composite with a pre-load of about 20 and $50 \mathrm{~N}$ cyclic preload. Filtek Supreme is considered as a better material than various other composites as water immersion test up to 1 year showed better results. Nanoclusters showed better reinforcement property, improved strength and reliability, irrespective of the environment conditions than hybrid and microfill. This is due to the silane infiltration in nanoclusters enhanced the damage tolerating capacity of the material

\section{CONCLUSIONS}

It has been proven by various studies that nanoparticles have improved mechanical properties and wear resistance. It has improved aesthetic output of the restoration by retaining the gloss and high translucency level of the restoration. High strength and radio opacity in the dental adhesive restoration have improved the longevity of the restoration.

\section{REFERENCES}

[1] Kirk RE, Othmer DF, Kroschwitz J. Nanotechnology: Encyclopedia of chemical technology. $4^{\text {th }}$ edn. New York: Wiley 1998;Suppl Volume:397-437.

[2] Kuhn E. Power Metallurgy ASM Handbook. ASM International, Materials Park, OH. Vol. 7. 1984: p. 56-70.

[3] Hiemenz PC. Principles of colloid and surface chemistry. New York, NY: Marcel Dekker Publishing Company, 1997.

[4] Moisescu C, Vroom Z, Dewith G. Synthesis of nanoapetite crystals, Phophorus. Res Bull 2002;13:193-6.

[5] Kong L, Gao Y, Cao W, et al. Preparation and characterization of nano-hydroxyapaetite /chitosan composite scaffolds. J Biomed Mater Res A $2005 ; 75 \mathrm{~A}(2): 275-82$.

[6] Liu C, Han Z, Czernuszka JT. Gradient collagen/nanohydroxyapatite composite scaffold: development and characterization. Acta Biomater 2009;5(2):661-9.

[7] Atai M, Solhi L, Nodehi A, et al. PMMA-grafted nanoclay as novel filler for dental adhesives. Dent Mater 2009;25(3):339-47. 
[8] Fortin D, Vargas MA. The spectrum of composite: new techniques and materials. J Am Dent Assoc 2000;Suppl 131:26S-30S.

[9] Denehy GE. A direct approach to restore anterior teeth. Am J Dent 2000;13(Spec No)55D-59D.

[10] Mitra SB, Wu D, Holmes BN. An application of nanotechnology in advanced dental materials. J Am Dent Assoc 2003;134(10):1382-90.

[11] Wu D, Kolb BU. Dental composites containing nanofillers and related methods. US patent 7 2008;393:882.

[12] Windisch MS, Zhang X, Rusin RP, et al. Clustered particle dental fillers. US patent 6 2004;730:156.

[13] Swift EJ. Ask the experts - Nanocomposites. J Esthet Restor Dent 2005;17(1):3-4.

[14] Gao F, Culbertson BM. Evaluation of multi metthacrylate -POSS for potential organic-inorganic hybrid dental restorative materials Polum. Prep Div Chem Am Chem Soc 2000;41:580-1.

[15] Mozner N, Klapdohr S. Nanotechnology for dental composites. Int J Nanotechnol 2004;1(1/2):130-56.

[16] Soh MS, Yap AUJ, Sellinger A. Methacrylate and epoxy functionalized nanocomposites based on silsesquioxane cores for use in dental applications. Eur Polym J 2007;43(2):315-27.

[17] Vollenweider M, Brunner TJ, Knecht S, et al. Remineralization of human dentin using ultrafine bioactive glass particles. Acta Biomater 2007;3(6):93643.

[18] Xu HH, Weir MD, Sun L. Nanocomposites with Ca and $\mathrm{PO}_{4}$ release: effects of reinforcement, dicalcium phosphate particle and silanization. Dent Mater 2007;23(12):148291.

[19] Xu HH, Moreau JL, Sun L, et al. Strength and fluoride release characteristics of a calcium fluoride based dental Nanocomposite. Biomaterials 2008;29(32):4261-7.

[20] Li L, Pan H, Tao J, et al. Repair of enamel by using hydroxyapetite nanoparticles as building blocks. J Mat Chem 2008;18(34):4079-84.

[21] Mitra S. Glass ionomers and related filling materials. In: Dhuru VB, edr. Contemporary dental materials. New Delhi, India: Oxford University Press 2004: p. 66-80.

[22] Blaes J. Pearls for your practice. Dent Econ 2007;97(9):154-6.

[23] Falsafi A, Madsen VJ, Mitra SB, et al. A novel nanoionomeric restorative with improved polish and wearresistance. J Dent Res 2008;87(Special Issue B: Abstract no 0244).
[24] Bui HT, Falsafi A, Mitra S, et al. Fluoride release of a new nano-ionomer restorative material. J Dent Res 2008;87 (Special Issue A: Abstract No 0987).

[25] Oxman JD, Falsafi A, Mitra S, et al. Improved polish, wear -resistance and esthetics of nano-ionomer restorative materials. J Dent Res 2008;87(Special Issue C: PEF IADR Abstract No 0039).

[26] Craig BD, Kolb BU, Oxman JD, et al. Use of nanoparticles to adjust refractive index of dental composites. US Patent 7,090, 721, 2006.

[27] Moshaverinia A, Ansari S, Movasaghi Z, et al. Modification of conventional glass-ionomer cements with $\mathrm{N}$-vinylpyrrolidone containing polyacids, nanohydroxy and fluorapetite to improve mechanical properties. Dent Mater 2008;24(10):1381-90.

[28] Prentice LH, Tyas MJ, Burrow MF. The effect of ytterbium fluoride and barium sulphate nanoparticles on the reactivity and strength of glass-ionomer cement. Dent Mater 2006;22(8):746-51.

[29] Davidson RS, Kolb BU, Anderson DB, et al. Zirconia particles. US Patent 7 2007;241:437.

[30] Silikas N, Masouras K, Satterthwaite J, et al. Effect of nanofillers in adhesive and aesthetic properties of dental resin-composites. Int J Nano \& Biomater 2007;1(2):11627.

[31] Heintze SD, Cavalleri A, Forjanic M, et al. A comparison of three different method for the quantification of the in vitro wear of dental materials. Dent Mater 2006;22(11):1051-62.

[32] De Gee AJ, Pallav P, Davidson CL. Effect of abrasion medium on wear of stress -bearing composites and amalgam in -vitro. J Dent Rest 1986;65(5):654-8.

[33] Wu D, Holmes BN, Mitra SB, et al. Wear resistance and mechanical properties of novel dental nanocomposites. J Dent Res 2002;81(Special Issue A-37 Abstract no 64).

[34] Wu D, Jones VW, Mitra SB, et al. Correlation of wear and surface roughness of dental materials. J Dent Res 2004;85(Special Issue A: Abstract no 3274).

[35] Cha HS, Lee YK, Lim BS, et al. Evaluation of wear resistance of dental resin composites with a 3D profilometer. J Biomed Mater Res B Appl Biomater 2004;71(2):414-20.

[36] Curtis AR, Palin WM, Fleming GJ, et al. The mechanical properties of nanofilled resin-based composites: characterizing discrete filler particles and agglomerates using a micromanipulation technique. Dent Mater 2009;25(2):180-7. 